\title{
Józef Dudek
}

Some remarks on distributive groupoids

Czechoslovak Mathematical Journal, Vol. 31 (1981), No. 3, 451-456

Persistent URL: http://dml.cz/dmlcz/101759

\section{Terms of use:}

(C) Institute of Mathematics AS CR, 1981

Institute of Mathematics of the Czech Academy of Sciences provides access to digitized documents strictly for personal use. Each copy of any part of this document must contain these Terms of use.

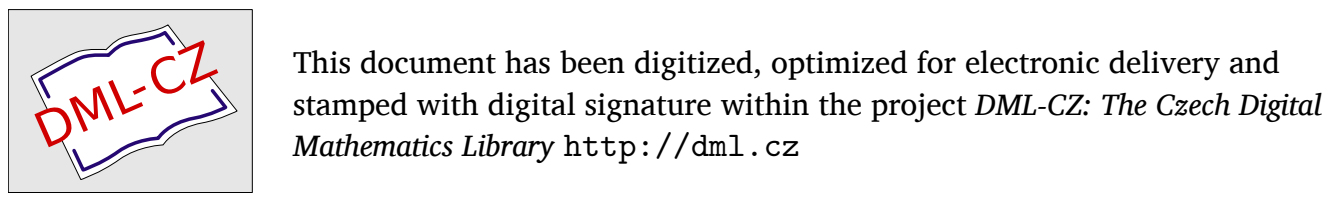




\title{
SOME REMARKS ON DISTRIBUTIVE GROUPOIDS
}

\author{
JózeF DUDEK, Wrocław
}

(Received June 20, 1979)

0. Introduction. A groupoid $(G, \circ)$ is said to be distributive if it satisfies $(x y) z=$ $=(x z)(y z)$ and $x(y z)=(x y)(x z)$ for all $x, y, z \in G$. If the groupoid satisfies the first of the above laws, then it is called right-distributive and if the second one, then it is called left-distributive. Our terminology and notations are those of [5] and [9].

In this note we give a characterization for distributive groupoids with algebraic constants (Theorem 1) and a characterization theorem for idempotent distributive groupoids with at most two essentially binary algebraic operations (polynomials) (Theorem 2). The variety of all distributive groupoids is denoted by $D$.

\section{EXAMPLES}

1.1. Nil-semigroups. A semigroup $(S,$.$) is said to be an n$-nil-semigroup if $x_{1} \ldots$ $\ldots x_{n}=0$ for a fixed element $0 \in S$ and all $x_{1}, \ldots, x_{n} \in S$. Denote by $S_{n}$ the variety of all $n$-nil-semigroups. It is easy to see that $S_{3}$ is properly contained in $D$. The name "nil-semigroup" was proposed to me by B. Gleichgewicht.

1.2. Diagonal semigroups. Let $T$ be the variety of all idempotent semigroups $(G,$. with $x y z=x z$ for all $x, y, z \in G$ (see [4], [11]). These groupoids will be called diagonal semigroups. Of course, $T \subset D$.

Let us say that a groupoid $(G, x \circ y)$ is dual with a given groupoid $(G, x y)$, if $x \circ y=y x$. If $K$ is a class of groupoids, then $K^{*}$ denotes the class of dual groupoids from $K$. Of course, if $x y=y x$ then $K^{*}=K$.

1.3. n-groupoids. Let $P_{1}$ be the class of all idempotent semigroups with $x y z=x z y$. This class was considered in [7] and [12]. Note that $P_{1}$ and $P_{1}^{*}$ are subvarieties of $D$. Indeed, let us show that $P_{1} \subset D$. We have $(x z)(y z)=((x z) y) z=x(z y z)=$ $=x y z(z)=x y z$. Analogously we prove the left-distributive law.

Let $P_{2}$ denote the variety of all idempotent groupoids which satisfy $(x y) z=y z$, $x(y z)=y(x z), x(x y)=y$, and let $P_{3}$ be the variety of all groupoids which are idem- 
potent and which satisfy $x(x y)=x y$, while the other two identities are the same as for the class $P_{2}$. It is not difficult to prove that $P_{i} \subset D$ and $P_{i}^{*} \subset D$ for $i=2,3$. These groupoids are considered in [2], [8] and [12] and are completely described in [12]. In [8] such groupoids are called $n$-groupoids (the same for their duals).

1.4. Semilattices and medial groupoids. Of course, the class of all semilattices (idempotent commutative semigroups) is a subvariety of the variety $D$. The same we have for the class $M$ of all idempotent commutative and medial groupoids (the medial law for groupoids means $(x y)(u v)=(x u)(y v))$. The class $M$ is considered in [3] and $[6]$.

1.5. Commutative Steiner quasigroups. A distributive groupoid $(G,$.$) is said to be$ a commutative Steiner quasigroup if it is commutative and $(x y) y=x$ for all $x, y \in G$ (see [1]). This class is considered in [3]. As is shown in [14] there exists a Steiner commutative quasigroup which is nonmedial. In [3] it is proved that any medial commutative Steiner quasigroup is a member of the variety $\operatorname{HSP}\left(\left(\{0,1,2\}, 2 x+{ }_{3} 2 y\right)\right)$ and every member of this variety is a medial commutative Steiner quasigroup.

1.6. Noncommutative Steiner quasigroups. In [10] the following class of groupoids $(G,$.$) is considered, namely, all groupoids which are idempotent and satisfy (x y) z=$ $=(z y) x$ and $(x y) x=y$. In [10] also a characterization for these groupoids is given. Observe that if an idempotent groupoid $(G,$.$) satisfies the above identities,$ then it is a noncommutative distributive groupoid. Indeed, let as check the rightdistributive law. We have $(x z)(y z)=((y z) z) x=((z z) y) x=(z y) x=(x y) z$. Suppose now that a $x=b$ for $a, b \in G$, then $x=(a x) a=b a$ and since the groupoid is cancellative we infer that $(G,$.$) is a distributive quasigroup. It is also easy to see$ that if card $G \geqq 2$ then $(G,$.$) is noncommutative. The above variety of groupoids$ leads us to the following definition: a groupoid $(G,$.$) is called a noncommutative$ Steiner quasigroup if it is distributive and satisfies $(x y) x=y$ and $x(x y)=y x$. Denote by $Q$ the variety of all noncommutative Steiner quasigroups.

\section{MAIN RESULTS}

In this section we prove two characterization theorems for some distributive groupoids.

Theorem 1. A distributive groupoid contains an algebraic constant if and only if it is a three-nil-semigroup.

Proof. As was mentioned in 1.1 every three-nil-semigroup with 0 is a distributive groupoid for which 0 is an algebraic constant. Let us suppose that $(G,$.$) is a dis-$ 
tributive groupoid with an algebraic constant 0 . To prove that $(G,$.$) is a three-nil-$ semigroup we use the formula of [9]. First we prove that for every distributive groupoid $(G,$.$) we have A^{(1)}(G,)=.\left\{x, x^{2}, x^{3}\right\}$, where $A^{(n)}(\mathfrak{H})$ denotes the set of all $n$-ary algebraic operations of an algebra $\mathfrak{A}$. For this definition and others used here see [9]. To prove that $A^{(1)}(G,)=.\left\{x, x^{2}, x^{3}\right\}$ we use the distributive laws and the formula from [9] for the set $A^{(n)}(\mathfrak{P})$ for a given algebra $\mathfrak{A}=(A, F)$, namely, $A^{(n)}=A^{(n)}(\mathfrak{I})=\bigcup_{k=0}^{\infty} A_{k}^{(n)}(\mathfrak{A})$, where $A_{0}^{(n)}(\mathfrak{Y})=\left\{e_{1}^{(n)}, \ldots, e_{n}^{(n)}\right\}$ and $e_{i}^{(n)}\left(x_{1}, \ldots, x_{n}\right)=x_{i}$ for $i=1, \ldots, n$ and $A_{k+1}^{(n)}(\mathfrak{A})=A_{k}^{(n)}(\mathfrak{Y}) \cup\left\{f\left(f_{1}, \ldots, f_{m}\right): f_{j} \in A_{k}^{(n)}(\mathfrak{U}), f \in F \quad\right.$ and $j=1, \ldots, m\}$. In our case $A^{(1)}=\bigcup_{k=0}^{\infty} A_{k}^{(1)}$, where $A_{0}^{(1)}=\{x\}$. We have $A_{1}^{(1)}=\left\{x, x^{2}\right\}$ and $A_{2}^{(1)}=\left\{x, x^{2}, x^{2} x, x x^{2}, x^{2} x^{2}\right\}=\left\{x, x^{2}, x^{3}\right\}$. Hence $x^{2} x=(x x) x=x^{2} x^{2}=$ $=(x x)(x x)=x x^{2}$. Now the proof follows by induction on $k$. Suppose that $A_{k}^{(1)}=\left\{x, x^{2}, x^{3}\right\}$. Take $A_{k+1}^{(1)}=A_{k}^{(1)} \cup\left\{x^{3} x, x x^{3}, x^{2} x^{2}, x^{2} x^{3}, x^{3} x^{2}, x^{3} x^{3}\right\}$. Hence $x^{3} x=\left(x^{2} x\right) x=x^{3} x^{2}=\left(x x^{2}\right) x^{2}=\left(x x^{2}\right)(x x)=x\left(x^{2} x\right)=x x^{3}=\left(x^{2} x\right) x^{2}=$ $=\left(x^{2} x^{2}\right)\left(x x^{2}\right)=x^{3} x^{3}=\left(x^{2} x\right)\left(x^{2} x\right)=x^{2} x^{2}=x^{3}$ and $x^{2} x^{3}=x^{2}\left(x^{2} x\right)=\left(x^{2} x^{2}\right)$. . $\left(x^{2} x\right)=x^{3} x^{3}=x^{3}$. We get $A_{k+1}^{(1)}=A_{k}^{(1)}$.

Thus we infer that $A^{(1)}=\left\{x, x^{2}, x^{3}\right\}$. But 0 is an algebraic constant in the groupoid $(G,$.$) , therefore there exists an algebraic operation f\left(x_{1}, \ldots, x_{n}\right)$ such that $f\left(x_{1}, \ldots, x_{n}\right)=0$ and hence $f(x, \ldots, x)=0$. This means that in the groupoid the identity $x=0$ or $x^{2}=0$ or $x^{3}=0$ holds. The first case says that the groupoid is a one - element groupoid and therefore it also is a three-nil-semigroup. If a distributive groupoid $(G,$.$) satisfies x^{2}=0$, then it satisfies also $x^{3}=0$. Indeed, $x^{3}=$ $=x^{2} x^{2}=00=0$. So, let us assume that $(G,$.$) satisfies x^{3}=0$. Then we have $0 x=x^{3} x=x x^{3}=x 0=x^{3}=0$ and $(x y) z=(x z)(y z)=((x z) y)((x z) z)=$ $=((x z) y)\left((x z)\left(z^{2}\right)\right)=((x z) y)\left(\left(x z^{2}\right)\left(z^{3}\right)\right)=((x z) y)\left(\left(x z^{2}\right) 0\right)=((x z) y) 0=0$.

Analogously, one can prove that $x(y z)=0$ for all $x, y, z \in G$. The proof of Theorem 1 is complete.

Remark. An example of a three-nil-semigroup $(G,$.$) with x^{2}=0$ can be obtained in the following way. Let $(G, \circ)$ be a nilpotent group of class 2 and take $(G,$.$) , where$ $x y=x^{-1} \circ y^{-1} \circ x \circ y$ for $x, y \in G$. Then $(G,$.$) is a three-nil-semigroup. However,$ there are three-nil-semigroups (see [13]) for which $x^{2}$ is not an algebraic constant.

Theorem 2. Let $(G,$.$) be an idempotent distributive groupoid with at most two$ essentially binary algebraic operations. Then one of the following possibilities occurs:

(1) $(G,$.$) is a semilattice,$

(2) $(G,$.$) is a diagonal semigroup,$

(3) $(G,$.$) is an n-groupoid,$

(4) $(G,$.$) is a commutative Steiner quasigroup,$ 
(5) $(G,$.$) is a noncommutative Steiner quasigroup,$

(6) $(G,$.$) is dual to an n-groupoid or (\mathrm{G}, \circ)$ is dual to a noncommutative Steiner quasigroup.

This theorem can be regarded as a characterization theorem for idempotent distributive groupoids with $\omega_{2} \leqq 2$. To prove this theorem we need some lemmas. For a given groupoid $(G,$.$) we agree to write x y^{n}$ instead of $(\ldots((x y) y) \ldots) y$, where $n \geqq 1$.

Lemma 1. If $(G,+)$ is idempotent commutative and nontrivial (card $G \geqq 2$ ), then $x+n y \neq y$ for all $n$.

Proof. Let $(G,+)$ be an idempotent commutative and non-one-element groupoid. Contrary to the lemma let us assume that the groupoid satisfies $x+n y=y$ for some $n$ and all $x, y \in G$. Let $m$ be the smallest number such that $x+m y=y$ holds in $(G,+)$. Putting in this identity $y+(m-1) x$ for $y$ we get $y+(m-1) x=$ $=(x+((y+(m-1) x)+(m-1)(y+(m-1) x)=((y+(m-1) x)+x)+$ $+(m-1)(y+(m-1) x)=(y+m x)+(m-1)(y+(m-1) x)=x+$ $+(m-1)(y+(m-1) x)=(x+(y+(m-1) x))+(m-2)(y+(m-1) x)=$ $=((y+(m-1) x)+x)+(m-2)(y+(m-1) x)=(y+m x)+(m-2)$. $.(y+(m-1) x)=x+(m-2)(y+(m-1) x)=\ldots=x+(y+(m-1) x)=$ $=(y+(m-1) x)+x=y+m x=x$.

So we get $x+(m-1) y=y$ for all $x, y \in G$ which contradicts the minimality of $m$.

Lemma 2. There is no idempotent commutative distributive groupoid $(G,+)$ for which $\omega_{2}(G,+)=2$.

Proof. Consider an algebraic operation $x+2 y$. Because of Lemma 1, one can assume that $x+2 y$ depends on $x$. If $x+2 y=x$ then the groupoid is a commutative Steiner quasigroup and for such non-trivial groupoids (as can easily be checked) we have $\omega_{2}=1$. Now assume that $x+2 y$ is essentially binary. Since $\omega_{2}(G,+)=2$ we infer that $x+2 y$ is symmetric, i.e., $x+2 y=y+2 x$. Using the last identity we have $x+2 y=(x+2 y)+(x+2 y)=(x+2 y)+(y+2 x)=$ $=((x+y)+y)+((x+y)+x)=(x+y)+(x+y)=x+y$.

It is easy to see that in this case $\omega_{2}(G,+)=1$ provided card $G \geqq 2$, a contradiction.

Lemma 3. If $(G,$.$) is an idempotent distributive groupoid with \omega_{2}(G,)=$.1 , then it is either a semilattice or a commutative Steiner quasigroup.

Proof. By the assumption and Lemma 1 we infer that the groupoid $(G, \circ)$ satisfies $x y^{2}=x$ or $x y^{2}=x y$. If the first case occurs then the groupoid is a commutative Steiner quasigroup. Assume now that $x y^{2}=x y$. Then we have $(x y) z=(x z)(y z)=$ 
$=(x(y z))(z(y z))=(x(y z))((y z) z)=(x(y z))(y z)=x(y z)$. This proves that $(G,$. is a semilattice. The proof of the lemma is complete.

Lemma 4. An idempotent distributive groupoid $(G,$.$) is a diagonal semigroup$ if and only if it satisfies $(x y) x=x$.

Proof. If a groupoid $(G,$.$) is a diagonal semigroup, then it is distributive idem-$ potent and $(x y) x=x$ (see 1.2 of Chapter 1$)$. Let now $(G,$.$) be idempotent distributive$ and $(x y) x=x$. Then we have $x=x(y x), x(x y)=((x y) x)(x y)=x y$ and $(y x) x=$ $=(y x)(x(y x))=y x$. Applying these facts we get $(x y) z=(x z)(y z)=((x z) y)$. $.((x z) z)=((x z) y)(x z)=x z$ and hence $x(y z)=(x y)(x z)=x(x z)=x z$, which proves that $(G,$.$) is a diagonal semigroup.$

Lemma 5. If $(G,$.$) is idempotent distributive and \omega_{2}(G,) \leqq$.2 and $(x y) x=y$, then it is either a commutative Steiner quasigroup or a noncommutative Steiner quasigroup.

Proof. If $x y=y x$ then the groupoid is a commutative Steiner quasigroup since $x=(y x) y=(x y) y=x y^{2}$. Assume now that $x y \neq y x$ and consider a binary polynomial $x y^{2}$. Since $y=(x y) x=x(y x)$ we infer that $(G,$.$) is cancellative and$ since $\omega_{2}(G,) \leqq$.2 , it is enough to examine the following identities $x y^{2}=x$ and $(x y) y=y x$ because otherwise the groupoid is trivial. If the first case occurs then we have $y x=y\left(x y^{2}\right)=y((x y) y)=x y$, a contradiction. If $(x y) y=y x$ in the groupoid then one has $y(y x)=: y((x y) y)=x y$ and hence $(G,$.$) is a noncommutative$ Steiner quasigroup.

Lemma 6. If an idempotent distributive groupoid $(G,$.$) satisfies \omega_{2}(G,) \leqq$. and $(x y) x \in\{x y, y x\}$, then it is either a semilattice or an n-groupoid.

Proof. First of all, assume that $(x y) x=x y$. Then we have $x(y z)=(x y)(x z)=$ $=((x y) x)((x y) z)=(x y)((x y) z)$. If $x(x y)=y$, then we get $x(y z)=z$ and hence $x=x(y x)=(x y) x=x y=x(y y)=y$ and the groupoid in this case is oneelement. Suppose now that $x(x y)=x$. Then $x(y z)=(x y)((x y) z)=x y$ and $(x y) z=$ $=(x z)(y z)=(x z) y$. So, the groupoid $\left(G\right.$, .) satisfies $x^{2}=x,(x y) z=(x z) y$ and $x(y z)=x y$. Since $\omega_{2}(G,) \leqq$.2 and card $G \geqq 2$, we infer that $(x y) y=x$ or $(x y) y=x y$ in the groupoid. Thus the groupoid is an $n$-groupoid. For example, let us prove that in such groupoids $(x y) y \neq y$ if card $G \geqq 2$. Indeed, if $(x y) y=y$, then using $x(y z)=x y$ we get $x y=x((x y) y)=x(x y)=x x=x$ and hence $y=$ $=(x y) y=x y=x$, a contradiction.

Assume now that $x(x y)=x y$. Then we have $x(y z)=(x y)(x z)=((x y) x)$. $.((x y) z)=(x y)((x y) z)=(x y) z$ and since $(x y) x=x y$ we get $x y z=x z y z=$ $=x z y$ which proves that $(G,$.$) is a semigroup that is an n$-groupoid. If $(x y) x=x y$ holds it remains to consider yet the case when $x(x y)=y x$. In this case we have 
$x(y z)=(x y)(x z)=((x y) x)((x y) z)=(x y)((x y) z)=z(x y)$ and hence $x y=$ $=x(y y)=y(x y)=(y x) y=y x$ which proves that $(G,$.$) is a semilattice. To$ complete the proof of the lemma, assume that $(x y) x=y x$. In this case the proof runs as above with the difference that we consider the operation $(x y) y$ and start from the identity $(x y) z=(x z)(y z)=(x(y z))(z(y z))=(x(y z))((z y) z)=(x(y z))$. . $(z y)$. The proof is complete.

Proof of Theorem 2. Let $(G,$.$) be an idempotent distributive groupoid with$ $\omega_{2}(G,) \leqq$.2 . Hence $\omega_{n}(\mathfrak{Q})$ (see [9]) is the number of all essentially $n$-ary algebraic operations of an algebra Therefore in our case $\omega_{2}(G,) \leqq$.2 means that $x y=x$ or $x y=y$ or $\omega_{2} \in\{1,2\}$. It is easy to see that the variety of all groupoids for which $x y=x$ (or dually $x y=y$ ) is a subvariety of the variety $T$ (see 1.2). Assume now that $1 \leqq \omega_{2}(G,) \leqq$.2 . This means that the fundamental operation is essentially binary. If the groupoid $(G,$.$) is commutative, then the proof follows from Lemmas 2$ and 3, if it is noncommutative then it follows from Lemmas 4, 5, and 6. Thus the proof of the theorem is complete.

\section{References}

[1] W. Belousov: Introduction in the theory of quasigroups and loops, Moscow (1967), (in Russian).

[2] J. Dudek: The number of algebraic operations in an idempotent groupoid, Cooloq. Math. 21. (1970), 169-177.

[3] J. Dudek: Medial groupoids and Mersenne numbers, to appear in Fund. Math.

[4] T. Evans: Products of points - some simple algebras and their identities, Amer. Math. Monthly Vol. 74, no 4. April, 1967.

[5] G. Gratzer: Universal Algebra, D. von Norstrand Company, 1968.

[6] J. Ježek and T. Kepka: The lattice of varieties of commutative abelian distributive groupoids, Algebra Univ. 5 (1975) 225-237.

[7] N. Kimura and M. Yamada: Note on idempotent semigroups II, Proc. of Jap. Acad., 34 (1958), 110-112.

[8] A. Kisielewicz: The number of algebraic operations in idempotent algebras, to appear in Algebra Univ.

[9] E. Marczewski: Independence and homomorphisms in abstract algebras, Fund. Math. 50 (1961), 45-61.

[10] A. Mitsche and H. Werner: On groupoids representable by linear spaces over finite fields, Arch. Math. Vol. 24 (1973), 14-20.

[11] J. Plonka: Diagonal algebras, Fund. Math. 50 (1966), 309-321.

[12] J. Plonka: On algebras with $n$ district essentially $n$-ary operations, Algebra Univ. (1971), $73-74$.

[13] J. Plonka: On functionally uniform symmetrical algebras, Colloq. Math. 15 (1966), 181 to 187.

[14] J. Soublin: Etude algebrique de la notion de moyenne, J. Math. Pures et App. 50 (1977), $53-264$.

Author's address: Institute of Mathematics, University of Wrockaw, Wrocław, Poland. 